\title{
Particulate Matter from Electronic Cigarettes and Conventional Cigarettes: a Systematic Review and Observational Study
}

\author{
Esteve Fernández ${ }^{1,2,3} \cdot$ Montse Ballbè $^{1,2,4} \cdot$ Xisca Sureda $^{1,2,5} \cdot$ Marcela Fu $^{1,2,3}$. \\ Esteve Saltó $^{2,3,6}$ • Jose M. Martínez-Sánchez ${ }^{1,2,7}$
}

Published online: 9 October 2015

(C) Springer International Publishing AG 2015

\begin{abstract}
Objectives The aim of this study is to review the literature on the composition of aerosols from electronic cigarettes (ecigarettes) originated by human vaping and to describe the emission of particulate matter $\leq 2.5 \mu \mathrm{m}$ in diameter $\left(\mathrm{PM}_{2.5}\right)$ from conventional and e-cigarettes at home in real-use conditions.

Methods We conducted a systematic literature search in PubMed and Web of Science. We measured $\mathrm{PM}_{2.5}$ in four different homes: one from a conventional cigarette smoker, one from an e-cigarette user, and two from non-smokers.

Results The review identified eight previous investigations on the composition of aerosols from e-cigarettes originated by human vaping and indicated that emissions from ecigarettes can contain potential toxic compounds such as nicotine, carbonyls, metals, and organic volatile
\end{abstract}

This article is part of the Topical Collection on Air Pollution and Health

Esteve Fernández

efernandez@iconcologia.net

Montse Ballbè

mballbe@iconcologia.net

Xisca Sureda

xisca.sureda@gmail.com

Marcela $\mathrm{Fu}$

mfu@iconcologia.net

Esteve Saltó

esteve.salto@gencat.cat

Jose M. Martínez-Sánchez

jmmartinez@iconcologia.nets

1 Tobacco Control Unit, Cancer Prevention and Control Program, Institut Català d'Oncologia, WHO Collaborating Center for Tobacco Control, L'Hospitalet de Llobregat, Barcelona, Spain compounds, besides particulate matter. In the observational study, the $\mathrm{PM}_{2.5}$ median concentration was $9.88 \mu \mathrm{g} / \mathrm{m}^{3}$ in the e-cigarette user home and 9.53 and $9.36 \mu \mathrm{g} / \mathrm{m}^{3}$ in the smoke-free homes, with $\mathrm{PM}_{2.5}$ peaks concurrent with the e-cigarette puffs.

Conclusion Both the literature review and the observational study indicate that e-cigarettes used under real-conditions emit toxicants, including $\mathrm{PM}_{2.5}$. Further research is needed to characterize the chemicals emitted by different types of ecigarettes and to assess secondhand exposure to e-cigarette aerosol using biological markers.

Keywords Tobacco smoke pollution · Particulate matter . Electronic cigarette $\cdot$ E-cigarette $\cdot$ Electronic nicotine delivery system

2 Cancer Prevention and Control Group, Institut d'Investigació Biomèdica de Bellvitge (IDIBELL), L'Hospitalet de Llobregat, Barcelona, Spain

School of Medicine, Universitat de Barcelona, Barcelona, Spain

4 Addictions Unit, Institute of Neurosciences, Hospital Clínic de Barcelona, Barcelona, Spain

5 Department of Preventive Medicine, Universidad de Alcalá, Alcalá de Henares, Spain

6 Health Plan Directorate, Ministry of Health, Generalitat de Catalunya, Spain

Biostatistics Unit, Department of Basic Sciences, Universitat Internacional de Catalunya, Sant Cugat del Vallès, Spain 


\section{Introduction}

Electronic cigarettes, also called "e-cigarettes" or "e-cigs," are the most known electronic nicotine delivery system. An ecigarette is an electronic device commonly shaped like a cigarette and designed to vaporize a mixture of nicotine, propylene glycol, and other chemicals. The e-cigarette heats the mixture via a battery activated by puffing. Interest in e-cigarettes has been recently growing among smokers, manufacturers, including leading cigarette companies, and also among tobacco control health professionals, researchers, and advocates who are concerned with their potential risks at the individual and public health level.

Concern exists regarding the potential passive exposure to the aerosol exhaled by e-cigarette users, as their use has increased in indoor places, including those with tobacco smokefree bans [1]. Some studies show that the aerosol generated from e-cigarettes contains toxic compounds (such as volatile organic compounds, aldehydes, nitrosamines, polyaromatic hydrocarbons, glycols, and nicotine), although in lower amounts than conventional cigarettes $[2 \bullet, 3,4,5 \bullet]$. Some of them have analyzed e-cigarette emissions, mainly in controlled conditions $[1,5 \cdot, 6]$, and have found that e-cigarettes emit fine and ultrafine particles (also known as particulate matter). The objective of this manuscript is to systematically review the existing literature on secondhand exposure from ecigarette aerosol in humans under real-life or mimicked reallife conditions and to describe the emission of particulate matter of less than $2.5 \mu \mathrm{m}$ in diameter $\left(\mathrm{PM}_{2.5}\right)$ from e-cigarettes at home in real-life use conditions and compare it that of conventional cigarettes.

\section{Methods}

\section{Literature Review}

We performed systematic literature search in PubMed (US National Library of Medicine; http://www.pubmed.org) and in the Web of Science (using the Web of Science ${ }^{8}$ Core Collection WoS, Thomson Reuters; http://webofscience. com) in order to identify relevant literature. Three search topics were combined: (1) "electronic nicotine delivery systems/electronic cigarettes," combined the search terms ("electronic cigarette*" OR e-cigarette* OR e-cig* OR ecig* OR "electronic nicotine delivery system*" OR "electronic nicotine delivery device*"); (2) "vapour", combined the search terms (vapor* OR vapour* OR aerosol* OR emission*); and (3) secondhand exposure, combined the search terms (secondhand OR second-hand OR passive OR involuntar* OR expos* OR environmental OR pollution).

The last updated literature search was performed in January 27, 2015. We identified 90 different articles for screening (33 duplicated in both databases). After reviewing the titles and abstracts, we find eligible 31 (see Fig. 1 for details) and reviewed their full text. We finally included eight studies focused on the composition of aerosol from e-cigarettes originated by human vaping. The other 23 articles excluded focused on health effects of vaping $(n=2)$ or in the composition of the aerosol of e-cigarettes originated by "smoking machines" $(n=21)$, which were not the focus of this paper (Fig. 1).

\section{Observational Study}

We measured $\mathrm{PM}_{2.5}$ in real conditions in the homes of one conventional cigarette smoker, one e-cigarette user, and two non-smokers (smoke-free homes), who voluntarily agreed to participate in the study and signed an informed consent form. The research and ethics committee of the Bellvitge University Hospital provided ethical approval for the study protocol. The e-cigarette user and the non-smokers lived in totally smokefree homes with no known infiltration of tobacco smoke into them from outdoors from other apartments in the same block. The measurement was taken for $1 \mathrm{~h}$ while the users of ecigarette or conventional cigarette were smoking $2 \mathrm{~m}$ away from the monitor. During that time, the conventional smokers smoked three cigarettes, and the e-cigarette user made 42 puffs (ad libitum use) using an e-liquid containing $18 \mathrm{mg}$ of nicotine (the e-cigarette device was Tornado ${ }^{\mathrm{TM}}$ model, one of the first medium-sized vaporizers launched in 2010, and the ecigarette liquid brand was Totally Wicked ${ }^{\mathrm{TM}}$ ). We registered the time when conventional cigarettes were lighted and the every puff was done (both for conventional and e-cigarettes). The measurements of $\mathrm{PM}_{2.5}$ were performed with a TSI SidePak Personal Aerosol Monitor model AM510 (TSI Inc. Minnesota, USA), which uses a built-in sampling pump to draw air through the device where the particulate matter in the air scatters the light from a laser determining the amount of light scattering. The monitor was zero-calibrated prior to each use with a HEPA filter, according to the manufacturer's specifications, and was set to a 1-s sampling interval, and a $\mathrm{K}$ factor of 0.52 was applied to data [7]. We plotted the $60-\mathrm{s}$ averaged concentrations of $\mathrm{PM}_{2.5}$ during the 1-h measurements. We computed the median (and interquartile range (IQR)) $\mathrm{PM}_{2.5}$ concentrations by type of home.

\section{Results}

\section{Literature Search}

Most studies tried to replicate the human vaping in enclosed settings (rooms between 8 and $60 \mathrm{~m}^{3}$ ) under controlled conditions, except a typical observational study conducted in Spain $[8 \bullet \cdot$. The main methodological characteristics and 
Fig. 1 Flow diagram of information through the different phases of the systematic review. WoS Web of Science

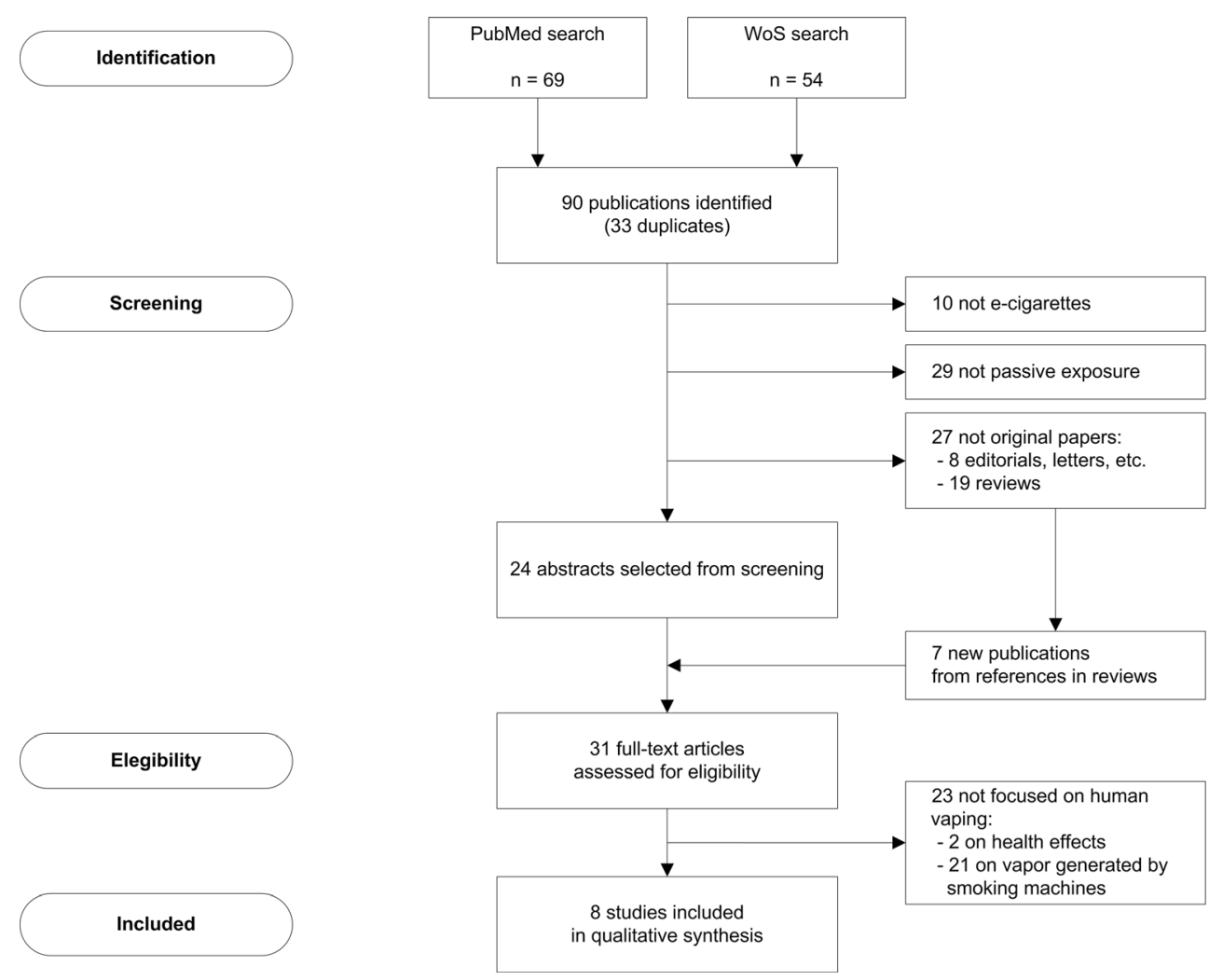

results are shown in Table 1. A study of the release of VOCs and fine and ultrafine particles from e-cigarettes under near-toreal-use conditions conducted in an experimental chamber with vapor produced by a volunteer who took six deep-lung puffs found an increase in fine particles, ultrafine particles, and VOCs after the use of an e-cigarette [2••]. The concentration of some aldehydes and other compounds were detected over the limit of determination as well as a high amount of 1,2propanediol and nicotine in the exhaled air. In an experimental study of secondhand aerosol exhaled by three volunteers, the median of the droplet size exhaled by the e-cigarette users were $0.34 \mu \mathrm{m}$ in e-cigarettes with nicotine and $0.29 \mu \mathrm{m}$ in the e-cigarettes without nicotine [9], indicating no difference in the particle diameter of the e-cigarettes with or without nicotine. In the investigation of emissions of particulate matter and ultrafine particles generated by e-cigarettes under mimicking real-life conditions in a $50-\mathrm{m}^{3}$ room furnished as an office where a volunteer used an e-cigarette with and without nicotine [6], total suspended particles emissions were systematically higher in vapor from e-cigarettes without nicotine $\left(11.6 \mu \mathrm{g} / \mathrm{m}^{3}\right)$ than from e-cigarettes with nicotine $(1.2 \mu \mathrm{g} /$ $\left.\mathrm{m}^{3}\right)$, but ultrafine particle concentrations were similar (641 particles $/ \mathrm{cm}^{3}$ among e-cigarettes without nicotine and 566 particles $/ \mathrm{cm}^{3}$ among e-cigarettes with nicotine). Two studies were performed to evaluate "the secondhand exposure to nicotine and other tobacco-related toxicants from e-cigarettes": the authors used five male volunteers (dual users of e- cigarettes and conventional tobacco cigarettes) to generate the vapor and found that e-cigarettes were a source of secondhand exposure to nicotine and $\mathrm{PM}_{2.5}$ but not to $\mathrm{CO}$ or VOCs, as compared to baseline (no emissions). An experimental study simulating a real-world scenario (café-like setting) [10•] assessed indoor concentrations of e-cigarette aerosol in terms of particulate matter and other compounds. During the vaping sessions, substantial amounts of 1,2-propanediol, glycerine, and nicotine were found in the gas phase, as well as high concentrations of $\mathrm{PM}_{2.5}$ (mean $197 \mu \mathrm{g} / \mathrm{m}^{3}$ ). In another experiment [11], the authors analyzed the particles and inorganic and organic compounds generated by the consumption of ecigarettes. The room mimicked a real-life setting under controlled conditions (a 48- $\mathrm{m}^{3}$ room where one volunteer used ecigarettes ad libitum). Organic and inorganic elements and metals were detected in the aerosol of e-cigarettes, including toxic metals (Ni, Zn, and Ag). The mass balance and distribution of water, glycerin, nicotine, phenolics, and carbonyls in exhaled e-cigarette aerosol was described in an experimental study with two disposable electronic cigarettes [12]. Total phenolics and carbonyls in exhaled e-cigarette aerosol were not significantly different than the amounts observed in exhaled breaths or air room samples. The only observational study available $[8 \bullet \bullet$ considered the exposure to e-cigarette aerosol during a week in the homes of a sample of five non-smokers non-exposed to secondhand smoke who lived with an e-cigarette user and 24 similar non- 
Table 1 Published papers on the composition of aerosols of electronic cigarettes originated by human vaping

\begin{tabular}{|c|c|c|c|c|}
\hline $\begin{array}{l}\text { Author, } \\
\text { publication } \\
\text { year (reference) }\end{array}$ & Design of study & Setting & $\begin{array}{l}\text { How emissions } \\
\text { were generated }\end{array}$ & Main finding(s) \\
\hline $\begin{array}{r}\text { Schripp et al. } \\
2013[2 \bullet \bullet\end{array}$ & Five controlled experiments & $\operatorname{Room}\left(48 \mathrm{~m}^{3}\right)$ & $\begin{array}{l}\text { A volunteering smoker } \\
\text { (experiment 1) and an } \\
\text { e-cigarette user } \\
\text { exhaling } \\
\text { one e-cigarette puff } \\
\text { (experiment 2) }\end{array}$ & $\begin{array}{l}\text { An increase in fine particles, ultrafine particles, and volatile } \\
\text { organic compounds were observed after the use of the } \\
\text { e-cigarette. } \\
\text { The concentration of some aldehydes and other compounds } \\
\text { were detected over the limit of determination. } \\
\text { The experiment revealed a high amount of 1,2-propanediol } \\
\text { in the exhaled air. Other main components were the carrier } \\
\text { substance 1,2,3-propanetriol, the flavoring source diacetin, } \\
\text { as well as traces of apple oil (3-methylbutyl-3- } \\
\text { methylbutanoate) and nicotine. }\end{array}$ \\
\hline $\begin{array}{l}\text { Bertholon et al. } \\
\quad 2013[9]\end{array}$ & $\begin{array}{l}\text { Experiment (six experiments, } \\
\text { three with nicotine and } \\
\text { three without nicotine) } \\
\text { simulated a real-world } \\
\text { scenario (cafe-like setting) }\end{array}$ & $\begin{array}{l}\text { Conference } \\
\text { room } \\
\left(40 \mathrm{~m}^{3}\right)\end{array}$ & Three volunteers & $\begin{array}{l}\text { The median of the droplet size exhaled by the e-cigarette users } \\
\text { were } 0.34 \mu \mathrm{m} \text { in electronic cigarettes with nicotine and } \\
0.29 \mu \mathrm{m} \text { in the e-cigarette without nicotine. }\end{array}$ \\
\hline $\begin{array}{l}\text { Ruprecht et al. } \\
\quad 2014[6]\end{array}$ & $\begin{array}{l}\text { Experiment simulating } \\
\text { real-life conditions }\end{array}$ & Homes & Three volunteer smokers & $\begin{array}{l}\text { PM and TSP emissions were systematically higher in } \\
\text { electronic cigarettes without nicotine. } \\
\text { PM emitted by electronic cigarettes without nicotine } \\
\text { were between } 3.5 \text { and } 9.9 \mu \mathrm{g} / \mathrm{m}^{3} \text {, depending on the } \\
\text { size of the particles. }\end{array}$ \\
\hline $\begin{array}{l}\text { Czogala } \\
\text { et al. } 2014[5 \cdot]\end{array}$ & $\begin{array}{l}\text { Experimental study with } \\
\text { two disposable electronic } \\
\text { cigarettes }\end{array}$ & & $\begin{array}{l}\text { Five volunteers (dual } \\
\text { e-cigarette/tobacco } \\
\text { smokers) }\end{array}$ & $\begin{array}{l}\text { Electronic cigarettes are a source of secondhand } \\
\text { exposure to nicotine and } \mathrm{PM}_{2.5} \text { but not to } \mathrm{CO} \text { or } \\
\text { volatile organic compounds (toluene), as compared } \\
\text { to baseline (no emissions). } \\
\text { Conventional cigarettes originated higher concentrations } \\
\text { of nicotine, } \mathrm{PM}_{2.5}, \mathrm{CO} \text {, and volatile organic } \\
\text { compounds (toluene, ethylbenzene, m,p-xylene, and } \\
\text { o-xylene), as compared to electronic cigarettes. }\end{array}$ \\
\hline $\begin{array}{l}\text { Schober } \\
\quad \text { et al. } 2014\left[10^{\bullet}\right]\end{array}$ & $\begin{array}{l}\text { Cross-sectional, } \\
\text { observational }\end{array}$ & & $\begin{array}{l}\text { Nine volunteer } \\
\text { occasional smokers }\end{array}$ & $\begin{array}{l}\text { During the vaping sessions substantial amounts of } \\
1,2 \text {-propanediol, glycerine, and nicotine were found in } \\
\text { the gas-phase, as well as high concentrations of } \mathrm{PM}_{2.5} \\
\text { (mean } 197 \mu \mathrm{g} / \mathrm{m}^{3} \text { ). } \\
\text { The concentration of putative carcinogenic PAH in indoor } \\
\text { air increased by } 20 \% \text { to } 147 \mathrm{ng} / \mathrm{m}^{3} \text {, and aluminum } \\
\text { showed a } 2.4-\text { fold increase. } \\
\text { PNC ranged from } 48,620 \text { to } 88,386 \text { particles } / \mathrm{cm}^{3} \text {, with } \\
\text { peaks at diameters } 24-36 \mathrm{~nm} \text {. }\end{array}$ \\
\hline $\begin{array}{l}\text { Saffari et al. } \\
\quad 2014 \text { [11] }\end{array}$ & Five controlled experiments & & $\begin{array}{l}\text { Three volunteer smokers } \\
\text { and a total of six } \\
\text { e-cigarette samples } \\
\text { (three with nicotine } \\
\text { and three without } \\
\text { nicotine) }\end{array}$ & $\begin{array}{l}\text { Black carbon and particle-phase PAHs were not detected in } \\
\text { e-cigarette's aerosol. } \\
\text { Emission rates of organic compounds as well as total } \\
\text { emission of inorganic elements and metals were detected } \\
\text { in electronic cigarettes. } \\
\text { There were also toxic metals (such as } \mathrm{Ni}, \mathrm{Zn} \text {, and } \mathrm{Ag} \text { ) in } \\
\text { e-cigarette's aerosol. } \\
\text { Secondhand particle-phase nicotine accounted for about } \\
0.02 \% \text { of the total nicotine generation and emission } \\
\text { during e-cigarette vaping. }\end{array}$ \\
\hline Long 2014 [12] & $\begin{array}{l}\text { Experiment (six experiments, } \\
\text { three with nicotine and } \\
\text { three without nicotine) } \\
\text { simulated a real-world } \\
\text { scenario (café-like setting) }\end{array}$ & & $\begin{array}{l}\text { Twenty electronic } \\
\text { cigarettes user } \\
\text { (maximum of } 99 \text { puffs) }\end{array}$ & $\begin{array}{l}\text { Distribution of exhaled e-cigarette aerosol showed the } \\
\text { composition was greater than } 99.9 \% \text { water and glycerin, } \\
\text { a small amount of nicotine }(<0.06 \%) \text {. } \\
\text { Total phenolics and carbonyls in exhaled e-cigarette aerosol } \\
\text { were not significantly different than the amounts observed } \\
\text { in exhaled breaths. }\end{array}$ \\
\hline $\begin{array}{l}\text { Ballbè et } \\
\quad \text { al. } 2014[8 \bullet \bullet]\end{array}$ & $\begin{array}{l}\text { Experiment simulating } \\
\text { real-life conditions }\end{array}$ & & $\begin{array}{l}\text { Real use of electronic } \\
\text { cigarettes during } \\
1 \text { week }\end{array}$ & $\begin{array}{l}\text { Airborne nicotine in e-cigarette users' homes was higher than } \\
\text { in control homes (smoke-free homes). }\end{array}$ \\
\hline
\end{tabular}

Adapted from Fernández E, Fu M, Martínez-Sánchez JM. Exposure to secondhand aerosol from electronic nicotine delivery systems: a systematic review. Barcelona: Institut Català d’Oncologia, WHO Collaborating Center for Tobacco Control; 2015 [18]

smokers in smoke-free and e-cigarette free homes. The median airborne nicotine concentrations in the homes of non-smokers exposed to e-cigarettes was 10-fold $\left(0.11 \mu \mathrm{g} / \mathrm{m}^{3}\right)$ higher than the nicotine concentration $\left(0.01 \mu \mathrm{g} / \mathrm{m}^{3}\right)$ in the control (smoke-free and e-cigarette free) homes. 


\section{Observational Study}

Figure 2 presents the real-time plots (moving average of $60 \mathrm{~s}$ ) of $\mathrm{PM}_{2.5}$ concentrations for $1 \mathrm{~h}$ in the four homes. The $\mathrm{PM}_{2.5}$ median concentration was $572.52 \mu \mathrm{g} / \mathrm{m}^{3}$ in the conventional cigarettes smoker's home (interquartile range (IQR) 431.08747.24). This concentration was significantly higher than the concentrations in the home of the e-cigarette user and the nonsmoker homes. The concentration in the home of the ecigarette user $\left(9.88 \mu \mathrm{g} / \mathrm{m}^{3}\right.$, IQR $\left.8.84-11.96\right)$ was similar to those in the non-smokers homes $\left(9.53 \mu \mathrm{g} / \mathrm{m}^{3}\right.$, IQR 8.32 10.50, and $9.36 \mu \mathrm{g} / \mathrm{m}^{3}$, IQR 8.84-10.40). While the $\mathrm{PM}_{2.5}$ medians in the e-cigarette user home and non-smokers smoke-free homes were similar, we noticed $\mathrm{PM}_{2.5}$ peaks concurrent with the e-cigarette puffs, as also shown in Fig. 2.

\section{Discussion}

The systematic review provides an overview of the few "reallife" studies on the seconhand exposureto aerosol of e-cigarettes. These studies indicate that emissions from e-cigarettes do contain potential toxic compounds such as nicotine, carbonyls, metals, and organic volatile compounds, besides particulate matter. While usually these compounds are generally at lower concentrations than those found in secondhand tobacco smoke, these findings made false the popular statement that ecigarette emissions are "only water vapor," or that they only include glycerin and propylene glycol beyond nicotine. The number of studies available and the types of e-cigarettes assessed is relatively small, and it is thus unknown if the chemicals and their concentrations vary markedly or not across different e-cigarette types. Moreover, whether secondhand exposure from e-cigarettes poses health risks at short- and long-term is still unknown, and needs further investigation.
Few studies have attempted to investigate e-cigarette aerosols in real-life conditions $[8 \bullet \bullet]$. In most of the papers $[2 \bullet \bullet, 5 \bullet, 6,9$, $10 \bullet, 11,12]$, "real-life conditions" refer to simulation of active vaping in a controlled room or chamber, by means of human volunteers actively vaping. Although this approach could serve to control for a number of variables by design, the conditions are so specific that generalization of results are far from satisfactory. Well conducted observational studies in true real conditions, in which the behavior of active vapers and bystanders is registered, together with a valid measurement of environmental markers and personal biomarkers of exposure, should offer new clues about the exposure to e-cigarette emissions.

We have found similar concentrations of $\mathrm{PM}_{2.5}$ in the smokefree homes and in the e-cigarette user homes, both under $10 \mu \mathrm{g} /$ $\mathrm{m}^{3}$, which is the threshold concentration for long-term exposures established in the Air Quality Guidelines of the World Health Organization [13]. This is in contrast to the $\mathrm{PM}_{2.5}$ concentrations in the conventional cigarette user's home, which were 58 times higher than in the e-cigarette user home. The air nicotine concentrations in the homes of smokers of conventional cigarettes were similar to the concentrations that have been observed in hospitality venues when smoking was allowed [14].

In our observational study, the particulate matter emissions from e-cigarette study were similar to those found in the smoke-free homes. We however observed $\mathrm{PM}_{2.5}$ peaks (over the $10 \mu \mathrm{g} / \mathrm{m}^{3}$ limit) concurrent with the e-cigarette puffs. This supports past observations that e-cigarettes emit particulate matter $[2 \bullet \bullet, 5 \bullet, 6,10 \bullet, 11]$. E-cigarettes produce an aerosol with fewer chemical components than those in conventional cigarettes because they do not require combustion, and hence, the temperature reached is lower than that in the conventional cigarettes, as shown in other studies $[3,15]$.

Some caution in the interpretation of the results of our observational study is needed, because they are based in the homes of four volunteers and only one vaper, using a specific

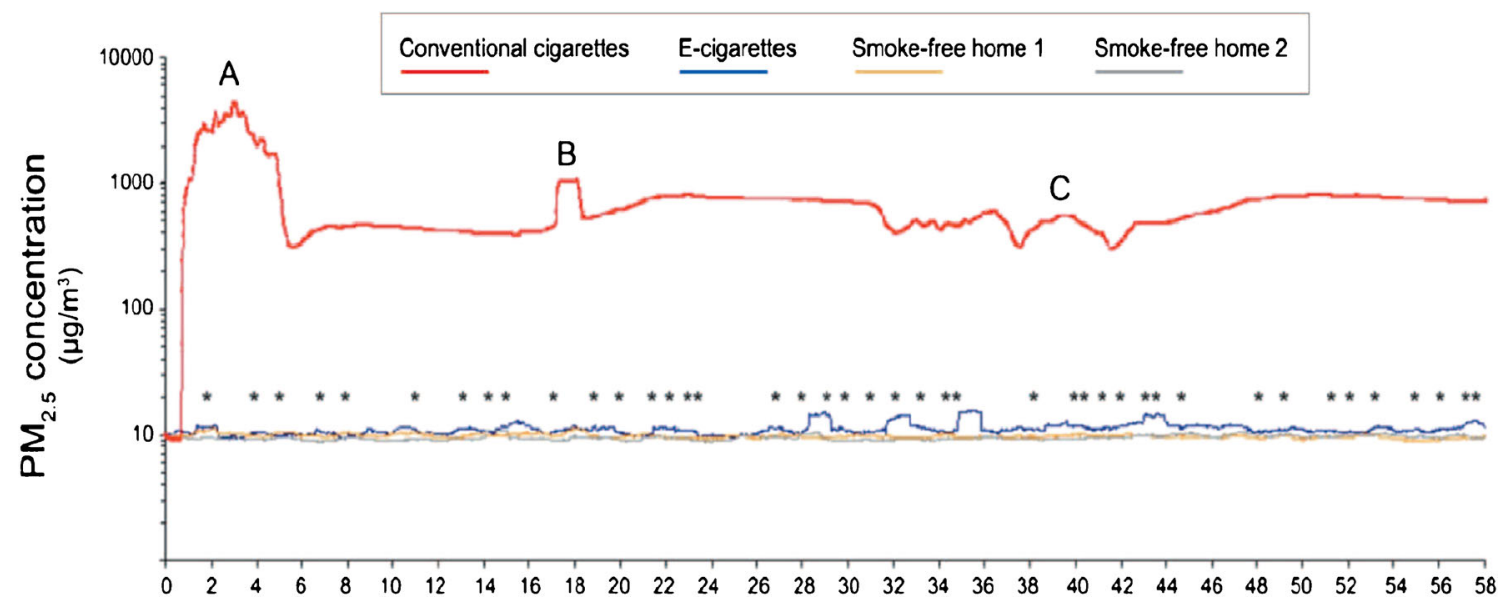

Fig. 2 Real-time $\mathrm{PM}_{2.5}$ concentrations (moving average of $60 \mathrm{~s}$ ) in the ecigarettes user's home, in a conventional cigarettes user's home, and in two smoke-free homes. Sixty-minute sampling while smoking or using e- cigarette. a One cigarette smoked for $6 \mathrm{~min}$. b One cigarette smoked for $7 \mathrm{~min}$. c One cigarette smoked for $5 \mathrm{~min}$. ${ }^{*}$ E-cigarette puff (42 puffs during the sampling period) 
type of vaporizer. Another potential limitation could be related to the possible differences (size and distribution) of the particulate matter from e-cigarettes and conventional cigarettes. An experimental study with aerosol from three e-cigarettes produced by a standard smoking machine [16] showed that the average particle number concentration and particle size of the aerosol from the e-cigarettes is comparable to that of the fresh mainstream tobacco burning cigarette smoke. However, differences among e-cigarette aerosols, due to differences in the type of devices (i.e., cig a likes, medium-sized vaporizers, and tank vaporizers or "mods") that operate at different voltages and temperatures are possible. Despite the potential limitations, our observational study is the first attempting to assess the emission of $\mathrm{PM}_{2.5}$ from e-cigarette vapor in real-life use conditions at home, with real e-cigarette and cigarette users and not smoking machines in a laboratory or controlled room, and a long time analyzed $(60 \mathrm{~min})$. As shown by the literature review, few studies have attempted to investigate e-cigarette aerosols in real-life or quasi-real-file conditions. In most of the papers, "real-life conditions" refer to simulation of active vaping in a controlled room or chamber, by means of human volunteers actively "vaping". Although this approach could serve to control for a number of variables by design, the conditions are so specific that generalization of results are far from satisfactory. In addition to further controlled experiments mimicking real-life conditions with using e-cigarette users to produce the aerosols, well designed and conducted observational studies in true real conditions, in which the behavior of not only active vapers but also bystanders is registered, together with a valid measurement of environmental markers and personal biomarkers of exposure, should offer complementary clues about the exposure to e-cigarette aerosols.

\section{Conclusions}

In addition to the literature results, our empirical results support that e-cigarette use in real conditions emit $\mathrm{PM}_{2.5}$, although these are notably lower than those from conventional cigarettes as also shown in previous studies. These results add new information to characterize secondhand exposure to ecigarette emissions and warrant further research using sensitive particle monitors to assess longer period of time [17]. Additional research is needed assessing these relevant chemicals and potential new ones across a variety of ecigarette devices as well as measuring personal biological markers among exposed people $[8 \bullet \bullet$.

Acknowledgments The authors wish to thank the volunteers that kindly collaborated in the observational study.

Contributors EF, MB, XS, MF, ES, and JMMS conceived the study. MB and JMMS conducted the fieldwork. EF, MF, and JMMS performed the literature search. EF, MB, MF, and JMMS analyzed the data. All authors contributed to data interpretation. EF drafted the manuscript, which was revised with substantial contributions from all the authors, who approved the final version. EF is the guarantor.

Funding This project was co-funded by the Instituto de Salud Carlos III, Subdirección General de Evaluación, Government of Spain and European Regional Development Fund (RTICC RD12/0036/0053, PI/ 081436, PI12/01114). The literature review is part of a comprehensive report prepared for (and supported by) the World Health Organization Tobacco Free Initiative (contract HQPND1409799).

\section{Compliance with Ethics Guidelines}

Conflict of Interest Esteve Fernández, Montse Ballbè, Xisca Sureda, Marcela Fu, Esteve Saltó, and Jose M. Martínez-Sánchez declare that they have no conflicts of interest.

Human and Animal Rights and Informed Consent Ethical approval for systematic reviews is not necessary and hence was requested. The ethics committee of the Bellvitge University Hospital provided ethical approval for the study protocol of the observational study including the information and written informed consent forms.

\section{References}

Papers of particular interest, published recently, have been highlighted as:

- Of importance

•- Of major importance

1. Convention Secretariat. WHO framework convention on tobacco control. Electronic nicotine delivery systems, including electronic cigarettes. Geneve: WHO; Publication No. FCTC/COP/5/13; 2012.

2.• Schripp T, Markewitz D, Uhde E, et al. Does e-cigarette consumption cause passive vaping? Indoor Air. 2013;23:25-31. This pioneer study showed for the first time that e-cigarette aerosols from real human vaping contain particulate matter, volatile organic compounds, aldehydes and propanediol.

3. McAuley TR, Hopke PK, Zhao J, et al. Comparison of the effects of e-cigarette vapor and cigarette smoke on indoor air quality. Inhal Toxicol. 2012;24:850-7.

4. Pellegrino RM, Tinghino B, Mangiaracina G, et al. Electronic cigarettes: an evaluation of exposure to chemicals and fine particulate matter (PM). Ann Ig. 2012;24:279-88.

5. Czogala J, Goniewicz ML, Fidelus B, et al. Secondhand exposure to vapors from electronic cigarettes. Nicotine Tob Res. 2014;16:65562. Controlled experiment with smokingmchine to evaluate the secondhand exposure to nicotine and other tobacco-related toxicants from e-cigarettes, showing that an emission of significant amounts of nicotine and PM2.5 but do not of CO.

6. Ruprecht AA, De Marco C, Pozzi P, et al. Comparison between particulate matter and ultrafine particle emission by electronic and normal cigarettes in real-life conditions. Tumori. 2014;100:e24-7.

7. Ruprecht AA, De Marco C, Boffi R, et al. Mass calibration and relative humidity compensation requirements for optical portable particulate matter monitors: the IMPASHS (impact of smoke-free policies in EU member states) WP2 preliminary results. Epidemiology. 2011;22:s206.

8.• Ballbè M, Martínez-Sánchez JM, Sureda X, et al. Cigarettes vs. ecigarettes: passive exposure at home measured by means of airborne marker and biomarkers. Environ Res. 2014;135C:76-80. 
Real-world observational study aimed at describing exposure to e-cigarettes aerosols using personal biomarkers (urinary and salivary cotinine) and environmental markers (airborne nicotine) from electronic cigarettes by exposed bystanders.

9. Bertholon J-F, Becquemin M, Roy M, et al. Comparison of the aerosol produced by electronic cigarettes with conventional cigarettes and the shisha. Rev Mal Respir. 2013;30:752-7.

10. Schober W, Szendrei K, Matzen W, et al. Use of electronic cigarettes (e-cigarettes) impairs indoor air quality and increases FeNO levels of e-cigarette consumers. Int J Hyg Environ Health. 2014;217:628-37. This study aimed at assessing indoor concentrations of e-cigarette aerosol in terms of particulate matter, particle number concentration, volatile organic compounds, polycyclic aromatic hydrocarbons, carbonyls, and metals.

11. Saffari A, Daher N, Ruprecht A, et al. Particulate metals and organic compounds from electronic and tobacco-containing cigarettes: comparison of emission rates and secondhand exposure. Environ Sci Process Impacts. 2014;16:2259-67.

12. Long GA. Comparison of select analytes in exhaled aerosol from ecigarettes with exhaled smoke from a conventional cigarette and exhaled breaths. Int J Environ Res Public Health. 2014;11:11177-91.
13. World Health Organization. Air quality guidelines, global update 2005. Geneva: WHO; 2006.

14. López MJ, Fernández E, Pérez-Ríos M, et al. Impact of the 2011 Spanish smoking ban in hospitality venues: indoor secondhand smoke exposure and influence of outdoor smoking. Nicotine Tob Res. 2013;15:992-6.

15. Goniewicz ML, Knysak J, Gawron M, et al. Levels of selected carcinogens and toxicants in vapour from electronic cigarettes. Inhal Toxicol. 2013;25:91-101.

16. Fuoco FC, Buonanno G, Stabile L, et al. Influential parameters on particle concentration and size distribution in the mainstream of ecigarettes. Environ Pollut. 2014;184:523-9.

17. Semple S, Apsley A, Maccalman L. An inexpensive particle monitor for smoker behaviour modification in homes. Tob Control. 2013;22:295-8.

18. Fernández E, Fu M, Martínez-Sánchez JM. Exposure to secondhand aerosol from electronic nicotine delivery systems: a systematic review. Barcelona: Institut Català d'Oncologia, WHO Collaborating Center for Tobacco Control; 2015. 